\title{
Anthropometric measurements and survival after a prostate cancer diagnosis
}

\author{
Megan S Farris ${ }^{1}$, Kerry S Courneya ${ }^{2}$, Karen A Kopciuk ${ }^{1,3,4}$, S Elizabeth McGregor ${ }^{3,5,6}$ and \\ Christine M Friedenreich ${ }^{\star 1,3,6}$ \\ ${ }^{1}$ Department of Cancer Epidemiology and Prevention Research, CancerControl Alberta, Alberta Health Services, Holy Cross \\ Centre, Calgary, $A B$, Canada; ${ }^{2}$ Faculty of Physical Education and Recreation, University of Alberta, Edmonton, AB, Canada; \\ ${ }^{3}$ Department of Oncology, Cumming School of Medicine, University of Calgary, Calgary, AB, Canada; ${ }^{4}$ Department of \\ Mathematics and Statistics, University of Calgary, Calgary, AB, Canada; Population, Public \& Indigenous Health, Alberta Health \\ Services, Calgary, $A B$, Canada and ${ }^{6}$ Department of Community Health Sciences, Cumming School of Medicine, University of \\ Calgary, Calgary, $A B$, Canada
}

Background: Evidence regarding the role of anthropometrics in prostate cancer survival is inconsistent. We examined the associations between anthropometric measures and survival outcomes.

\begin{abstract}
Methods: Men diagnosed with prostate cancer $(n=987)$ were recruited into a population-based case-control study between 1997 and 2000, then a prospective cohort study between 2000 and 2002, where anthropometric measurements (weight, height, body mass index, waist circumference, waist-hip ratio) were taken and participants were followed up to 19 years for survival outcomes. Cox proportional hazards were used to examine these associations.
\end{abstract}

Results: Survival analyses suggested no clear pattern of associations between post-diagnosis anthropometric measurements and all-cause mortality, prostate-specific mortality, first recurrence/progression or new primary cancer.

Conclusions: We did not find a significant trend relating anthropometrics to survival outcomes after prostate cancer diagnosis. Continued assessment of objective measurements of body composition over the life-course is warranted to determine true associations between anthropometrics and survival after prostate cancer.

Obesity and prostate cancer negatively impacts a large proportion of men in Canada. Since nearly $95 \%$ of prostate cancer survivors will survive at least 5 years after their diagnosis (Canadian Cancer Society's Advisory Committee on Cancer Statistics, 2016) and obesity rates continue to increase in Canada (Statistics Canada, 2014), it is of particular relevance for public health to determine how and if obesity is related to prostate cancer survival (Twells et al, 2014).

While there is increasing epidemiologic evidence providing insights into how different measures of anthropometrics affect prostate cancer survival (Cao and Ma, 2011; Meyer et al, 2015), examining multiple time-points and different anthropometric measures would be valuable to establish the role of anthropometrics in prostate cancer survival (Cao and Giovannucci, 2016; Yang et al, 2016). A recent dose-response meta-analysis, focused on body mass index (BMI) and prostate cancer-specific mortality, found pre-diagnosis but not post-diagnosis BMI was related to prostate cancer-specific mortality and no associations with allcause mortality (Zhong et al, 2016).

The objective of this study was to examine how different anthropometrics were associated with survival outcomes in prostate cancer survivors.

\section{MATERIALS AND METHODS}

Study population. Men diagnosed with prostate cancer were recruited to a population-based case-control study conducted between 1997 and 2000 in Alberta, Canada (Friedenreich et al, 2004) and were re-consented to a prospective cohort study and

*Correspondence: Dr C Friedenreich; E-mail: christine.friedenreich@albertahealthservices.ca

Received 25 July 2017; revised 9 November 2017; accepted 10 November 2017; published online 12 December 2017

(C) 2018 Cancer Research UK. All rights reserved 0007 - 0920/18 
followed for survival outcomes. The details of this study have been previously published (Friedenreich et al, 2016). In brief, participants were $<80$ years of age, diagnosed with incident prostate cancer (stage $\geqslant \mathrm{T} 2$ ) with no prior cancer diagnosis (except non-melanoma skin cancer) and able to speak English at initial enrolment. Ethics approval for this study was approved by the Health Research Ethics Board of Alberta - Cancer Committee and the Conjoint Health Research Ethics Board at the University of Calgary.

Data collection. At baseline (1997-2000) within six months of diagnosis, interviewers used standardised methods and calibrated weight scales to obtain current adiposity measurements from prostate cancer cases including: height, weight, waist circumference and hip circumference. At follow-up which occurred 2-3 years post-diagnosis in the prospective cohort study (2000-2002; $n=829$ ), interviewers assessed the same current anthropometric measurements as done objectively at baseline.

Other potentially relevant lifestyle risk factors and personal health history data were collected at baseline and updated at the first follow-up. Medical chart abstractions and vital status updates were performed to update information on treatment and prostate cancer status by the Alberta Cancer Registry and Cancer Surveillance. Altogether, there was over 19 years of follow-up for survival outcomes from baseline to 20 January 2017.
Statistical analysis. Descriptive characteristics of all anthropometric variables and covariates in the final models were determined. Anthropometric measurements were categorised based on conventional cutoffs: BMI (World Health Organization, 2006) (underweight and normal weight groups were combined because only one participant had a $\mathrm{BMI}<18$ ), waist circumference and waist-hip ratio (Consultation, 2008) or by quartiles: height, weight, weight gained since age 20 and weight change after diagnosis. Martingale residuals were calculated and graphed (Therneau et al, 1990) to confirm linearity prior to quartile categorisations of anthropometric variables (Harrell et al, 1988). The Schoenfeld residuals test was used to assess the proportional hazards assumption by testing the independence between these residuals and time (Schoenfeld, 1982). Cox proportional hazards were fit to produce adjusted hazard ratios and 95\% confidence intervals for all models. Competing risk Fine and Grey methods (Fine and Gray, 1999) were used to determine if competing risks influenced the prostate cancer-specific mortality models. Death from other causes (not prostate cancer-related) was classified as the competing risk. Models were fit while adjusting for the following variables a priori: age at diagnosis, stage, prostatectomy, initial hormone therapy treatment, initial radiation therapy treatment, prostate specific antigen levels at diagnosis, and post-diagnosis Charlson comorbidity index score. The same abovementioned

Table 1. Anthropometric measurements at baseline (within 6 months of diagnosis) in relation to all-cause mortality and prostate cancer-specific mortality in the Prostate Cohort Study in Alberta, Canada, 1997-2017 ( $n=987$ )

\begin{tabular}{|c|c|c|c|c|c|c|}
\hline \multirow[b]{2}{*}{ Anthropometric measurements } & \multicolumn{3}{|c|}{ All-cause mortality } & \multicolumn{3}{|c|}{ Prostate cancer-specific mortality } \\
\hline & $\begin{array}{c}\text { All-cause } \\
\text { deaths/cases }\end{array}$ & $\begin{array}{l}\text { Age-adjusted } \\
\text { HR }(95 \% \mathrm{Cl})\end{array}$ & $\begin{array}{c}\text { Multivariable } \\
\text { adjusted HR } \\
(95 \% \mathrm{Cl})^{\mathrm{a}}\end{array}$ & $\begin{array}{c}\text { Prostate } \\
\text { cancer deaths/ } \\
\text { cases }\end{array}$ & $\begin{array}{l}\text { Age-adjusted } \\
\text { HR }(95 \% \mathrm{Cl})\end{array}$ & $\begin{array}{c}\text { Multivariable } \\
\text { adjusted } \mathrm{HR} \\
(95 \% \mathrm{Cl})^{\mathrm{b}}\end{array}$ \\
\hline \multicolumn{7}{|l|}{ Height $(\mathrm{cm})^{c}$} \\
\hline$<169.6$ & $172 / 242$ & 1.0 & 1.0 & $65 / 242$ & 1.0 & 1.0 \\
\hline $169.6-<173.9$ & $176 / 251$ & $0.83(0.67-1.03)$ & $0.86(0.69-1.07)$ & $65 / 251$ & $0.82(0.58-1.16)$ & $0.99(0.68-1.42)$ \\
\hline $173.9-<178$ & $166 / 246$ & $0.86(0.69-1.08)$ & $0.87(0.69-1.09)$ & $62 / 246$ & $0.82(0.57-1.17)$ & $0.81(0.55-1.19)$ \\
\hline $178+$ & $142 / 248$ & $0.70(0.55-0.89)$ & $0.74(0.58-0.95)$ & $60 / 248$ & $0.71(0.49-1.04)$ & $0.77(0.52-1.15)$ \\
\hline \multicolumn{7}{|l|}{ Weight $(\mathrm{kg})^{\mathrm{d}}$} \\
\hline$<75.7$ & $179 / 248$ & 1.0 & 1.0 & $62 / 248$ & 1.0 & 1.0 \\
\hline $75.7-<84.4$ & $156 / 243$ & $0.79(0.63-0.98)$ & $0.84(0.67-1.06)$ & $61 / 243$ & $0.87(0.61-1.24)$ & $1.03(0.71-1.49)$ \\
\hline $84.4-<93.2$ & $154 / 249$ & $0.90(0.72-1.12)$ & $0.94(0.75-1.19)$ & $60 / 249$ & $0.90(0.63-1.29)$ & $0.94(0.64-1.37)$ \\
\hline $93.2+$ & $167 / 247$ & $1.10(0.88-1.39)$ & $1.08(0.85-1.36)$ & $69 / 247$ & $1.08(0.76-1.52)$ & $1.09(0.75-1.58)$ \\
\hline \multicolumn{7}{|c|}{ Weight gain from age 20 to current weight $(\mathrm{kg})^{d}$} \\
\hline$<5.8$ & $181 / 246$ & 1.0 & 1.0 & $73 / 246$ & 1.0 & 1.0 \\
\hline $5.8-<13.7$ & $153 / 245$ & $0.75(0.60-0.92)$ & $0.89(0.71-1.11)$ & $47 / 245$ & $0.57(0.39-0.83)$ & $0.80(0.55-1.17)$ \\
\hline $13.7-<21.6$ & $155 / 246$ & $0.77(0.62-0.96)$ & $0.85(0.68-1.06)$ & $63 / 246$ & $0.77(0.53-1.10)$ & $0.88(0.63-1.25)$ \\
\hline $21.6+$ & $167 / 250$ & $0.95(0.77-1.17)$ & $0.92(0.74-1.14)$ & $69 / 250$ & $0.85(0.57-1.28)$ & $0.94(0.67-1.31)$ \\
\hline \multicolumn{7}{|l|}{ BMI $\left(\mathrm{kg} \mathrm{m}^{-2}\right)$} \\
\hline$<25$ & $148 / 213$ & 1.0 & 1.0 & $53 / 213$ & 1.0 & 1.0 \\
\hline $25-<30$ & $316 / 493$ & $0.87(0.72-1.06)$ & $0.88(0.72-1.07)$ & $113 / 493$ & $0.85(0.62-1.18)$ & $0.79(0.57-1.10)$ \\
\hline $30+$ & $192 / 281$ & $1.13(0.91-1.40)$ & $1.09(0.88-1.36)$ & $86 / 281$ & $1.29(0.92-1.82)$ & $1.13(0.80-1.61)$ \\
\hline \multicolumn{7}{|l|}{ Waist circumference $(\mathrm{cm})$} \\
\hline$<94$ & $177 / 273$ & 1.0 & 1.0 & $72 / 273$ & 1.0 & 1.0 \\
\hline $94-<102$ & $175 / 279$ & $0.93(0.75-1.14)$ & $0.89(0.72-1.10)$ & $63 / 279$ & $0.84(0.60-1.18)$ & $0.76(0.54-1.07)$ \\
\hline $102+$ & $304 / 435$ & $1.17(0.97-1.41)$ & $1.07(0.88-1.29)$ & $117 / 435$ & $1.09(0.81-1.46)$ & $0.84(0.62-1.13)$ \\
\hline \multicolumn{7}{|l|}{ Waist-hip ratio $(\mathrm{cm})$} \\
\hline$<0.90$ & $98 / 167$ & 1.0 & 1.0 & $41 / 167$ & 1.0 & 1.0 \\
\hline $0.90-<0.95$ & $173 / 271$ & $1.10(0.85-1.40)$ & $0.98(0.76-1.26)$ & $69 / 271$ & $1.07(0.73-1.58)$ & $0.91(0.61-1.36)$ \\
\hline $0.95+$ & $385 / 549$ & $1.34(1.07-1.67)$ & $1.14(0.91-1.42)$ & $142 / 549$ & $1.17(0.82-1.65)$ & $0.92(0.64-1.32)$ \\
\hline \multicolumn{7}{|c|}{ 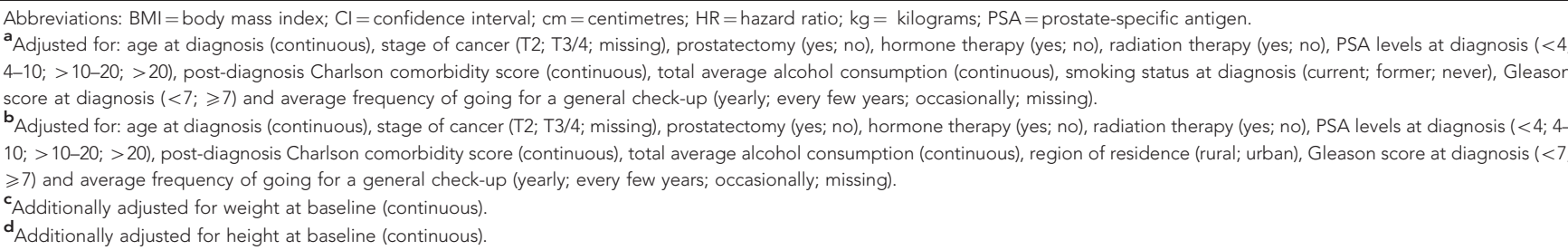 } \\
\hline
\end{tabular}


variables in addition to Gleason score at diagnosis, family history of cancer, total lifetime physical activity and smoking status were tested for interaction to determine any potential effect modification. Sensitivity analyses were performed to determine the influence of time to first recurrence for all survival models, treated as a timevarying covariate. In addition, deaths occurring in the first 2 years of follow-up were excluded to address reverse causation. Further, testing for potential confounding (through backwards elimination) was done for the following variables: total lifetime physical activity, total average alcohol consumption, average daily caloric intake, education level, smoking status diagnosis, family history of cancer, region of residence, Gleason score at diagnosis, and how often (on average) participants went for a general check-up in their lifetime prior to diagnosis of prostate cancer.

All statistical analysis was performed in Stata (version13; StataCorp LP, College Station, TX, USA), assumptions (proportional hazards and linearity) were met and all $P$-values were assessed at $\alpha<0.05$.

\section{RESULTS}

A flowchart of participants' progress in the study has been reported (Friedenreich et al, 2016) and their final vital status to 20 January 2017 is presented. Briefly, the final analytic samples for the baseline anthropometric measurements analysis and the post-diagnosis first follow-up analysis were 987 and 829, respectively. Of the total sample $(n=987), 655$ men died from any cause, 252 died of prostate cancer and 437 had a first recurrence/progression or new primary cancer. Most characteristics did not significantly differ between the total sample and those who had an outcome
(Supplementary Table 1). Differences in baseline characteristics between the total sample $(n=987)$, those who survived $2-3$ years post-diagnosis $(n=829)$ and those who survived $5-7$ years postdiagnosis were not significant (data not shown).

Effect modification was not present and therefore results are presented for the overall study population only. Further, age-adjusted models were compared to multivariable adjusted models in final tables showing some evidence of confounding. Time to recurrence, death within the first 2 years $(n=49)$ and first recurrence/ progression or new primary cancer within the first 2 years $(n=92)$ did not statistically change our results. Results were comparable between Fine and Grey competing risk and Cox proportional hazards models for prostate cancer-specific mortality; therefore, Cox proportional hazards multivariable models were presented. In the baseline and follow-up analyses, there was no consistent pattern for how anthropometric measures were associated with all-cause mortality or prostate cancer-specific mortality (Tables 1 and 2) or first recurrence/progression or new primary cancer (Supplementary Tables 2 and 3). In addition, tests for trend in all analyses were not statistically significant (data not shown).

\section{DISCUSSION}

In this survival analysis, we found that all-cause mortality, prostate cancer-specific mortality and first recurrence/progression or new primary cancer after a prostate cancer diagnosis were not consistently related to the battery of anthropometrics assessed shortly after diagnosis (at baseline) and 2-3 years post-diagnosis. In fact, a protective effect of increased or high anthropometric measurements was suggested in some of our results. The biological

Table 2. Anthropometric measurements in survivors 2-3 years post-diagnosis $(n=829)$ in relation to all-cause mortality and prostate cancer-specific mortality in the Prostate Cohort Study in Alberta, Canada, 1997-2017

\begin{tabular}{|c|c|c|c|c|c|c|}
\hline \multirow[b]{2}{*}{$\begin{array}{l}\text { Anthropometric } \\
\text { measurements }\end{array}$} & \multicolumn{3}{|c|}{ All-cause mortality } & \multicolumn{3}{|c|}{ Prostate cancer-specific mortality } \\
\hline & $\begin{array}{c}\text { All-cause } \\
\text { deaths/cases }\end{array}$ & $\begin{array}{c}\text { Age-adjusted HR } \\
(95 \% \mathrm{Cl})\end{array}$ & $\begin{array}{c}\text { Multivariable adjusted } \\
\text { HR }(95 \% \mathrm{Cl})^{\mathrm{a}}\end{array}$ & $\begin{array}{l}\text { Prostate cancer } \\
\text { deaths/cases }\end{array}$ & $\begin{array}{c}\text { Age-adjusted HR } \\
(95 \% \mathrm{Cl})\end{array}$ & $\begin{array}{l}\text { Multivariable adjusted } \\
\text { HR }(95 \% \mathrm{Cl})^{\mathrm{b}}\end{array}$ \\
\hline \multicolumn{7}{|l|}{ Weight $(\mathrm{kg})^{c}$} \\
\hline$<77.3$ & $143 / 206$ & 1.0 & 1.0 & $45 / 206$ & 1.0 & 1.0 \\
\hline $77.3-<85.9$ & $133 / 208$ & $0.90(0.70-1.14)$ & $0.87(0.68-1.11)$ & $40 / 208$ & $0.82(0.53-1.27)$ & $0.78(0.50-1.23)$ \\
\hline $85.9-<95.1$ & $125 / 209$ & $0.91(0.71-1.17)$ & $0.95(0.73-1.23)$ & $41 / 209$ & $0.84(0.54-1.31)$ & $0.88(0.55-1.39)$ \\
\hline $95.1+$ & $127 / 206$ & $1.09(0.84-1.43)$ & $1.02(0.78-1.33)$ & $59 / 206$ & $1.33(0.86-2.04)$ & $1.17(0.74-1.86)$ \\
\hline \multicolumn{7}{|c|}{ Weight change from baseline to follow-up $(\mathrm{kg})^{c, d}$} \\
\hline$<-4.5$ & $123 / 204$ & $0.91(0.71-1.17)$ & 0.89 (0.69-1.15) & $60 / 204$ & $0.76(0.52-1.12)$ & 0.73 (0.49-1.10) \\
\hline$-4.5-<-1.6$ & $129 / 205$ & $0.87(0.68-1.12)$ & $0.90(0.70-1.15)$ & $47 / 205$ & $0.61(0.41-0.92)$ & $0.69(0.45-1.06)$ \\
\hline$-1.6-<1.2$ & $137 / 214$ & 1.0 & 1.0 & $40 / 214$ & 1.0 & 1.0 \\
\hline $1.2+$ & $139 / 206$ & $1.01(0.79-1.29)$ & $1.05(0.82-1.35)$ & $38 / 206$ & $0.64(0.42-0.97)$ & $0.77(0.50-1.19)$ \\
\hline \multicolumn{7}{|l|}{ BMI $\left(\mathrm{kg} \mathrm{m}^{-2}\right)$} \\
\hline$<25$ & $98 / 140$ & 1.0 & 1.0 & $31 / 140$ & 1.0 & 1.0 \\
\hline $25-<30$ & $256 / 401$ & $0.84(0.67-1.06)$ & $0.83(0.66-1.05)$ & $82 / 401$ & $0.84(0.56-1.27)$ & $0.73(0.48-1.11)$ \\
\hline $30+$ & $174 / 288$ & $0.93(0.73-1.20)$ & $0.90(0.70-1.17)$ & $72 / 288$ & $1.08(0.71-1.66)$ & $0.97(0.63-1.50)$ \\
\hline \multicolumn{7}{|c|}{ Waist circumference $(\mathrm{cm})$} \\
\hline$<94$ & $92 / 153$ & 1.0 & 1.0 & $34 / 153$ & 1.0 & 1.0 \\
\hline $94-<102$ & $126 / 195$ & $1.08(0.83-1.42)$ & $1.03(0.79-1.35)$ & $35 / 195$ & $0.79(0.50-1.27)$ & $0.68(0.42-1.09)$ \\
\hline $102+$ & $310 / 482$ & $1.15(0.91-1.46)$ & $1.06(0.83-1.34)$ & $116 / 482$ & $1.11(0.76-1.63)$ & $0.90(0.61-1.32)$ \\
\hline \multicolumn{7}{|c|}{ Waist-hip ratio $(\mathrm{cm})$} \\
\hline$<0.90$ & $46 / 74$ & 1.0 & 1.0 & $21 / 74$ & 1.0 & 1.0 \\
\hline $0.90-<0.95$ & $83 / 145$ & $1.01(0.71-1.45)$ & 1.05 (0.73-1.50) & $24 / 145$ & $0.57(0.32-1.03)$ & $0.58(0.32-1.05)$ \\
\hline $0.95+$ & $399 / 611$ & $1.21(0.89-1.64)$ & $1.13(0.83-1.55)$ & $140 / 661$ & $0.83(0.52-1.31)$ & $0.71(0.44-1.13)$ \\
\hline \multicolumn{7}{|c|}{$\begin{array}{l}\text { Abbreviations: } \mathrm{BMI}=\text { body mass index; } \mathrm{Cl}=\text { confidence interval; } \mathrm{cm}=\text { centimetres; } \mathrm{HR}=\text { hazard ratio; } \mathrm{kg}=\text { kilograms. } \\
\text { a Adjusted for: age at diagnosis (continuous), stage, prostatectomy, hormone therapy, radiation therapy, PSA levels at diagnosis, post = diagnosis Charlson comorbidity score, total average } \\
\text { alcohol consumption (continuous), region of residence and } \mathrm{Gleason} \text { score at diagnosis. } \\
\text { bAdjusted for: age at diagnosis (continuous), stage of cancer ( }(\mathrm{T} 2 ; \mathrm{T} 3 / 4 ; \text { missing), prostatectomy (yes; no), hormone therapy (yes; no), radiation therapy (yes; no), PSA levels at diagnosis ( }<4 \text {; } \\
4-10 ;>10-20 ;>20 \text { ), post-diagnosis Charlson comorbidity score (continuous), family history of cancer (yes; no) and Gleason score at diagnosis }(<7 ; \geqslant 7) \text {. } \\
{ }^{c} \text { Additionally adjusted for height at baseline (continuous). } \\
{ }^{d} \text { Additionally adjusted for weight at baseline (continuous). }\end{array}$} \\
\hline
\end{tabular}


plausibility of a protective association between obesity-related anthropometric measures and survival after prostate cancer may be attributable to sarcopenia (Walston, 2012), since the average age at diagnosis of our study population was 67.3 years. Men with higher anthropometrics, especially abdominal girth, may have more fat energy storage to fight off their disease, relative to men with lower anthropometric measurements. However, we also cannot rule out that we may be underpowered to detect an association or the chance of Type I error. The epidemiologic evidence is inconsistent and somewhat limited with respect to the role of different measures of anthropometrics after prostate cancer diagnosis, especially longterm survival (Cao and Giovannucci, 2016; Yang et al, 2016) with most of the literature presenting null findings and/or using selfreported data. Our study also did not find an association between anthropometrics and prostate cancer survival, despite examining several objective anthropometric measurements from participants.

There are study limitations that need to be considered. First, while we did have objective anthropometric measures, total body composition measured directly using a dual-energy $\mathrm{x}$-ray or adiposity using a computerised tomography scan is preferred to eliminate potential measurement bias. In addition, we may have been underpowered, specifically in the prostate-cancer specific mortality models with only 252 events, the detection of effect modification and the stratified analyses; therefore, future research in a larger sample size should consider if important prognostic factors influence these relations. In addition, while we did combine first progression/recurrence or new primary cancers as one outcome, to increase clinical relevance, future studies should analyse these outcomes separately to appropriately determine if there are meaningful differences between these outcomes. Generalisability of our results may be limited to prostate cancer survivors with $\geqslant \mathrm{T} 2$ cancer at diagnosis and not all prostate cancer survivors. Further, including intentional weight change would add an additional dimension of interest that would permit distinguishing between possible sarcopenia and intentional weight loss in this population.

Strengths of the study include the prospective study design, the long follow-up period of up to 19 years and the repeated anthropometric assessments post-diagnosis. Specifically, we were able to demonstrate that weight change after prostate cancer diagnosis does not lead to poorer prognosis, which is emerging in the literature (Caan and Kroenke, 2017). In addition, we tested for competing risks when evaluating prostate cancer-specific mortality, which appropriately isolates this outcome and is especially important with prostate cancer, considering these older men die more frequently from other causes.

Altogether, there was not a clear trend relating anthropometric measures to survival or risk of recurrence/progression or new primary cancer after prostate cancer. Continued future assessment of life-course adiposity/anthropometric patterns may provide more insight than static estimations of adiposity, specifically when related to mortality and other cancer outcomes.

\section{ACKNOWLEDGEMENTS}

Study coordination was done by Aleata Ryhorchuk and Sana Fakih. Interviewers for this study were Jodi Parrotta, Linda Davison, Pearl Cooke, Nicole Slot, Carol-Anne Zawalykut, Catherine Munro. Data entry was done by Carla Quesnel and chart abstraction by Yvonne LeBlanc and Sarah MacLaughlin. Dr Steven J Angyalfi provided clinical expertise regarding prostate cancer treatment and care.

This work was supported by the Canadian Institute for Health Research (2004-2007; grant number MOP-67217); the National
Cancer Institute of Canada with funds from the Canadian Cancer Society (2000-2004; grant number 011004); and the Alberta Cancer Board-Research Initiative Program (2004-2007; grant number 4570). Christine M Friedenreich held career awards from Alberta Innovates, Health Solutions and Alberta Cancer Foundation, Kerry S Courneya was supported by the Canada Research Chairs Program and S Elizabeth McGregor by the Alberta Heritage Foundation for Medical Research.

\section{CONFLICT OF INTEREST}

The authors declare no conflict of interest.

\section{REFERENCES}

Caan BJ, Kroenke CH (2017) Next steps in understanding the obesity paradox in cancer. Cancer Epidemiol Biomarkers Prev 26(1): 12.

Canadian Cancer Society's Advisory Committee on Cancer Statistics (2016) Canadian Cancer Statistics 2016. Canadian Cancer Society: Toronto, ON.

Cao Y, Giovannucci E (2016) Obesity and prostate cancer. Recent Results Cancer Res 208: 137-153.

Cao Y, Ma J (2011) Body mass index, prostate cancer-specific mortality, and biochemical recurrence: a systematic review and meta-analysis. Cancer Prev Res (Phila) 4(4): 486-501.

Consultation WE (2008) Waist circumference and waist-hip ratio. Report of a WHO Expert Consultation. World Health Organization: Geneva, pp $8-11$.

Fine JP, Gray RJ (1999) A proportional hazards model for the subdistribution of a competing risk. J Am Statist Assoc 94: 496-509.

Friedenreich CM, McGregor SE, Courneya KS, Angyalfi SJ, Elliott FG (2004) Case-control study of lifetime total physical activity and prostate cancer risk. Am J Epidemiol 159(8): 740-749.

Friedenreich CM, Wang Q, Neilson HK, Kopciuk KA, McGregor SE, Courneya KS (2016) Physical activity and survival after prostate cancer. Eur Urol 70(4): 576-585.

Harrell Jr FE, Lee KL, Pollock BG (1988) Regression models in clinical studies: determining relationships between predictors and response. J Natl Cancer Inst 80(15): 1198-1202.

Meyer J, Rohrmann S, Bopp M, Faeh D (2015) Impact of smoking and excess body weight on overall and site-specific cancer mortality risk. Cancer Epidemiol Biomarkers Prev 24(10): 1516-1522.

Schoenfeld D (1982) Partial residuals for the proportional hazards regression model. Biometrika 69(1): 239-241.

Statistics Canada (2014) Overweight and obese adults (self-reported). Statistics Canada: Ottawa.

Therneau TM, Grambsch PM, Fleming TR (1990) Martingale-based residuals for survival models. Biometrika 77(1): 147-160.

Twells LK, Gregory DM, Reddigan J, Midodzi WK (2014) Current and predicted prevalence of obesity in Canada: a trend analysis. CMAJ open 2(1): E18-E26.

Walston JD (2012) Sarcopenia in older adults. Curr Opin Rheumatol 24(6): 623-627.

World Health Organization (2006) Global Database on Body Mass Index: BMI Classification. World Health Organization: Geneva.

Yang L, Drake BF, Colditz GA (2016) Obesity and other cancers. J Clin Oncol 34(35): 4231-4237.

Zhong S, Yan X, Wu Y, Zhang X, Chen L, Tang J, Zhao J (2016) Body mass index and mortality in prostate cancer patients: a dose-response metaanalysis. Prostate cancer Prostatic dis 19(2): 122-131.

This work is published under the standard license to publish agreement. After 12 months the work will become freely available and the license terms will switch to a Creative Commons AttributionNonCommercial-Share Alike 4.0 Unported License. 